“ (C) 2015 IEEE. Personal use of this material is permitted. Permission from IEEE must be obtained for all other uses, in any current or future media, including

reprinting/republishing this material for advertising or promotional purposes, creating new collective works, for resale or redistribution to servers or lists, or reuse of any copyrighted component of this work in other works." 


\title{
An Extension to the Filtered-x LMS Algorithm with Logarithmic Transformation
}

\author{
Marek Pawelczyk ${ }^{1}$, Witold Wierzchowski ${ }^{2}$, Lifu $\mathrm{Wu}^{3}$, Xiaojun Qiu ${ }^{4}$ \\ ${ }^{1,2}$ Institute of Automatic Control, Silesian University of Technology, Gliwice, Poland \\ marek.pawelczyk@polsl.pl,witold.wierzchowski@polsl.pl, \\ ${ }^{3}$ College of Electronic \& Information Engineering, Nanjing University of Information Science and Technology, Nanjing, China \\ wulifu@nuist.edu.cn, \\ ${ }^{4}$ Key Laboratory of Modern Acoustics and Institute of Acoustics, Nanjing University, Nanjing, China \\ xjqiu@nju.edu.cn
}

\begin{abstract}
Active control of impulsive noise has been of increasing interest due to high impact of such noise on humans. The algorithm with logarithmic transformation, developed by $\mathrm{Wu}$, et al. has been found particularly interesting. In this paper this idea is continued, and an extension to this algorithm is proposed to improve its convergence properties and allow for successful control if the noise has also another type of noise together with the impulses. A number of simulations are performed to validate the algorithm and compare it with algorithms leading in the literature. Additionally to simulated benchmark impulsive noises, real recordings are considered, which bring another insight into efficiency of the algorithms.
\end{abstract}

Keywords-Active noise control; impulsive noise; mixed noise; FxLogLMS; NSFxLogLMS.

\section{INTRODUCTION}

Active Noise Control (ANC) is usually explained by destructive interference between original primary noise and secondary sound generated by a control algorithm [1]. Most commonly used ANC algorithm is the Filtered-x LMS (FxLMS), thanks to its low computational complexity, robustness, good convergence properties and satisfactory performance [2]. However, there are several noise types, for which the FxLMS algorithm is not suitable. One of them is impulsive noise, for which FxLMS exhibits convergence problems or even can make the overall system unstable [8]. ANC for impulsive noise is thus particularly challenging [5].

A typical single-channel feed-forward ANC system is presented in Fig. 1. It consists of a reference signal $x(n)$, an error signal $\mathrm{e}(\mathrm{n})$, a primary path $\mathrm{P}(\mathrm{z})$, a secondary path $\mathrm{S}(\mathrm{z})$, and an adaptive control filter $\mathrm{W}(\mathrm{z}) ; z$ is a complex variable and the paths are assumed linear. The control filter can be presented as a tap weight vector of length $\mathrm{L}_{\mathrm{w}}$ :

$$
\mathbf{w}(n)=\left[\mathrm{w}_{0}(\mathrm{n}), \mathrm{w}_{1}(\mathrm{n}), \ldots, \mathrm{w}_{\mathrm{L}_{\mathrm{w}}-1}\right]^{\mathrm{T}} .
$$

$\hat{\mathrm{S}}(\mathrm{z})$ stands for the secondary path model. An update equation for the FxLMS algorithm is as follows:

$$
\mathbf{w}(n+1)=\mathbf{w}(n)+\mu e(n) \mathbf{x}_{\mathbf{s}}(n),
$$

The research has been supported by the National Science Centre, Poland, decision no. DEC-2012/07/B/ST7/01408. where $\mu$ is step size parameter responsible for convergence rate adjustment, and xs is a vector of filtered reference signal:

$$
\mathbf{x}_{\mathbf{s}}(\mathrm{n})=\left[\mathrm{x}_{\mathrm{s}}(\mathrm{n}), \mathrm{x}_{\mathrm{s}}(\mathrm{n}-1), \ldots, \mathrm{x}_{\mathrm{s}}\left(\mathrm{n}-\mathrm{L}_{\mathrm{w}}+1\right)\right]^{\mathrm{T}} \text {. }
$$

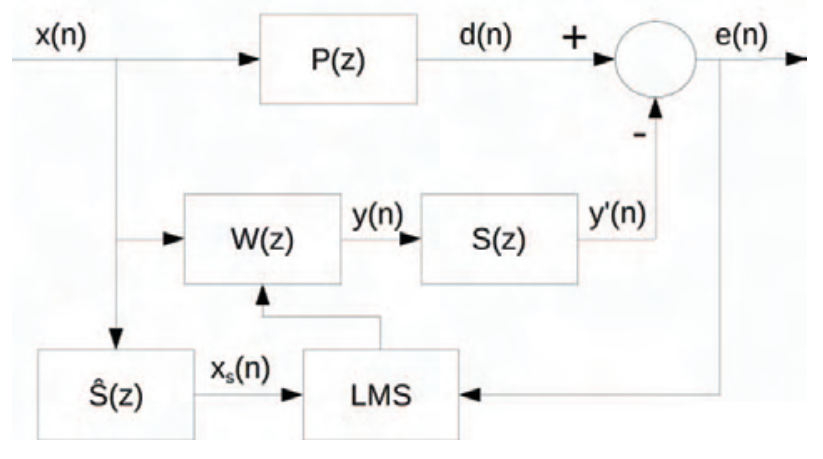

Fig. 1. Block diagram of a single-channel feedforward ANC system with the FxLMS algorithm.

Impulsive noise, due to its properties, is often described using symmetric $\alpha$-stable distribution with the following characteristic function:

$$
\varphi(t)=\exp \left(j a t-\gamma|t|^{\alpha}\right),
$$

where parameter $\alpha$ is referred to as a characteristic exponent (or stability parameter), and it is responsible for width of the tails, a is a location parameter and $\gamma$ is a scale parameter [3], [4]. Parameter $\alpha$ can vary from 0 to 2 , and its smaller values indicate a more impulsive character.

For acoustic impulsive noise, modeling with the standard distribution is used, i.e. parameters $\gamma=1$ and $a=0$. Probability distributions for several values of $\alpha$ are presented in Fig. 2 and an exemplary time plot for $\alpha=1.7$ is shown in Fig. 3. In case of signal obeying alpha stable distribution, only moments of order less than the characteristic exponent exist, what means that if $\alpha<2$ then the variance of the signal does not exist. Since optimization criterion of the standard FxLMS algorithm 
depends strongly on the error signal variance, it may become unstable for impulsive noise.

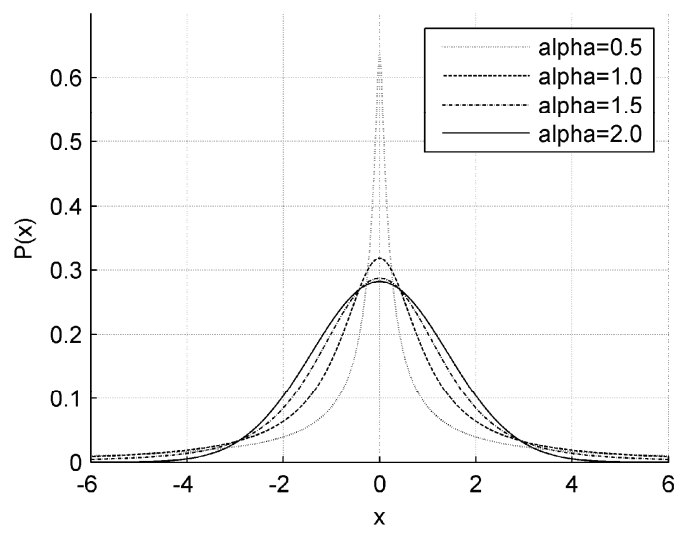

Fig. 2. Probability density functions for several values of the characteristic exponent.

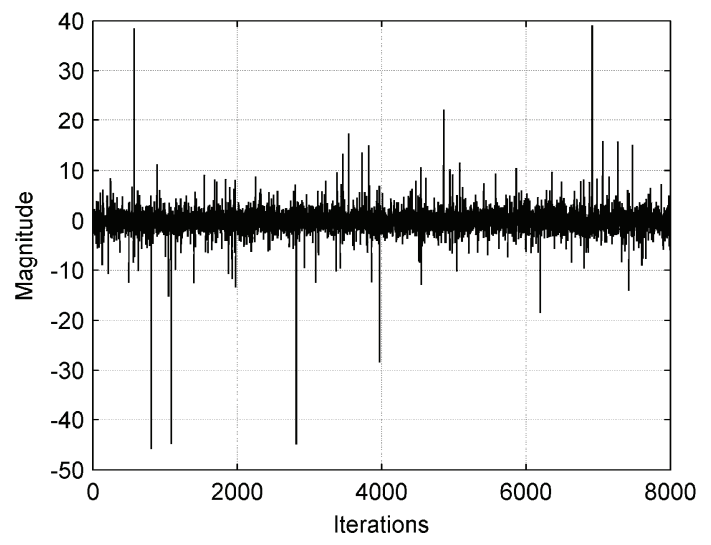

Fig. 3. Exemplary time plot for $\alpha$-stable process $\alpha=1.7$

\section{SOlutions For ACTIVE CONTROL OF IMPUlsive NoISE}

In this Section the Normalized FxLMS and Normalized FxLMP algorithms are presented. Moreover, several variations of the FxLMS algorithm are briefly described for reference.

Akhtar et al. [6] proposed Modified Normalized version of the FxLMS algorithm (MNFxLMS). It takes advantage of normalization of the step size, which was proposed for the LMS algorithm in [7]. Akhtar et al. modified the normalization factor to take into account not only energy of the reference signal, but also energy of the error signal, which may contain impulses if they have not been reduced yet. Those impulses might force the algorithms to correct the control filter in a wrong direction. Resulting update equation and normalization equation are then as follows:

$$
\begin{gathered}
\mathbf{w}(\mathrm{n}+1)=\mathbf{w}(\mathrm{n})+\mu(\mathrm{n}) \mathrm{e}(\mathrm{n}) \mathbf{x}_{\mathbf{s}}(\mathrm{n}), \\
\mu(\mathrm{n})=\frac{\tilde{\mu}}{\left\|\mathbf{x}_{\mathbf{s}}(\mathrm{n})\right\|_{2}^{2}+\mathrm{E}_{\mathrm{e}}(\mathrm{n})+\delta},
\end{gathered}
$$

where $E_{e}(n)$ is an estimate of the residual error signal:

$$
E_{e}(n)=\lambda E_{e}(n-1)+(1-\lambda) e^{2}(n),
$$

and $\lambda$ is a forgetting factor of value between 0.9 and 1 , $\left\|\mathbf{x}_{\mathbf{s}}(\mathrm{n})\right\|_{2}^{2}$ represents $\mathrm{L}_{2}$ norm of the filtered reference signal and $\delta$ is a small positive number added to avoid division by zero.

Since for alpha stable distributions, moments higher than characteristic exponent do not exist, a minimization criterion basing on lower order moment was proposed in [12]. The Filtered-x Least Mean $p$-Power algorithm (FxLMP) update equation is as follows:

$$
\mathbf{w}(n+1)=\mathbf{w}(n)+\mu p|e(n)|^{p-1} \operatorname{sgn}(e(n)) \mathbf{x}_{\mathbf{s}}(n),
$$

where the sign function is defined as:

$$
\operatorname{sgn}(e)=\left\{\begin{array}{r}
1, \text { e }>0 \\
0, e=0 \\
-1, e<0
\end{array}\right.
$$

Similarly to MNFxLMS, a normalization procedure was proposed by Akhtar et al. for the FxLMP algorithm, resulting in the Modified Normalized FxLMP (MNFxLMP) algorithm [4]. The update equation takes the following form:

$$
\mathbf{w}(\mathrm{n}+1)=\mathbf{w}(\mathrm{n})+\mu(\mathrm{n}) \mathrm{p}|\mathrm{e}(\mathrm{n})|^{\mathrm{p}-1} \operatorname{sgn}(\mathrm{e}(\mathrm{n})) \mathbf{x}_{\mathbf{s}}(\mathrm{n}),
$$

where

$$
\mu(\mathrm{n})=\frac{\tilde{\mu}}{\left\|\mathbf{x}_{\mathbf{s}}(\mathrm{n})\right\|_{\mathrm{p}}^{\mathrm{p}}+\mathrm{E}_{\mathrm{e}}(\mathrm{n})+\delta},
$$

and $\left\|\mathbf{x}_{\mathbf{s}}(\mathrm{n})\right\|_{\mathrm{p}}^{\mathrm{p}}$ represents the $p$-th norm of the filtered reference signal. It is assumed that $p$ should be smaller than the value of characteristic exponent of alpha stable distribution.

There is also a group of ANC algorithms, which uses modified reference and error signals. First algorithm from this group was proposed by Sun, et al. in [11]. The idea is to ignore samples of the reference signal, i.e. substitute them with 0 , if its magnitude is greater than some assumed value. An extension to this algorithm was proposed by Akhtar, et al. in [13], which neglects also samples with big magnitude in the error signal. Further improvement considered saturating reference and error signals at some level instead of ignoring them [10]. All of these algorithms show better robustness to impulsive noise than the FxLMS algorithm. However, these algorithms require proper selection of threshold parameters, which may not be possible in some applications. It has been shown in [6] that the normalized algorithms are faster than any algorithm from aforementioned group.

\section{LOGARITHMIC ALGORITHM, [3]}

$\mathrm{Wu}$, et al., [3], analyzed logarithmic transformation of the impulsive noise samples and concluded that the transformed 
process obeys the Gaussian distribution. Basing on these observations they proposed to minimize

$$
J(n)=\log ^{2}|e(n)|,
$$

instead of the squared error itself. With this approach, the spread of the minimized signal is compressed, and the algorithm is not forced to dramatically change parameters of the control filter in response to an impulse in the noise. This brings a high progress to impulsive noise control. Minimization of the above cost function leaded to deriving the FxLogLMS algorithm of the following control filter update equation [3]:

$$
w(n+1)=w(n)+\mu \operatorname{sgn}(e(n)) \frac{\log |e(n)|}{|e(n)|} x_{s}(n) .
$$

$\mathrm{Wu}$ et al. noticed that when the value of the error signal tends to zero, the logarithmic function tends to infinity. To cope with this problem they suggested to set $|e(n)|=1$ if $|e(n)|<1$, which resulted in stopping the control filter update for small values of the error signal (due to the logarithmic function). Impulsive noise control results demonstrated superiority of this algorithm over a number of other algorithms. However, no comparison between the FxLogLMS and the normalized algorithms was presented. Such comparison is presented further in this paper.

\section{Proposed AlgorithM}

Stopping control filter update in the FxLogLMS algorithm for $|e(n)|<1$ is an appropriate solution if only impulsive noise is concerned. However, for many active noise control applications like active device casings, there is another type of noise beyond impulses, due to working mechanisms or engines. It is thus beneficial to offer an algorithm, which could also successfully reduce that noise, in between the impulses as defined in [14]. Preliminary experiments have shown that the FxLogLMS algorithm might then behave less efficiently than the classical FxLMS algorithm. In this section, the idea of the FxLogLMS algorithm, originally developed in [3], is extended to offer a more general-purpose algorithm, appropriate for working in environments, where impulsive noise is accompanied by other noises. In order to improve stability and convergence speed of the algorithm, normalization of the step size parameter is introduced. This concept was used for the FxLMS algorithm in [6] and also for the FxLMP in [4]. Resulting update equation for Normalized FxLogLMS can be presented as:

$$
\begin{gathered}
w(n+1)=w(n)+\mu(n) \operatorname{sgn}(e(n)) \frac{\log |e(n)|}{|e(n)|} x_{s}(n) \\
\mu(n)=\frac{\tilde{\mu}}{\left\|x_{s}(n)\right\|_{2}^{2}+\delta} .
\end{gathered}
$$

Another modification to the FxLogLMS algorithm is adding a "switching" filter, which improves algorithm convergence rate for signals that are not purely impulsive or even non-impulsive at all. The idea is to switch between the Normalised FxLogLMS algorithm when error signal values are large, and the Normalised FxLMS, when error signal values are small. As for the FxLogLMS, $|\mathrm{e}(\mathrm{n})|=t$ is assumed as the threshold. Noting also that

$$
\frac{\operatorname{sgn}(e(n))}{|e(n)|}=\frac{1}{e(n)}
$$

the update equation can be written as:

$$
\begin{gathered}
\mathrm{w}(\mathrm{n}+1)= \\
\left\{\begin{array}{c}
\mathrm{w}(\mathrm{n})+\frac{\tilde{\mu}}{\left\|\mathrm{x}_{\mathrm{S}}(\mathrm{n})\right\|_{2}^{2}+\delta} \frac{\log |\mathrm{e}(\mathrm{n})|}{\mathrm{e}(\mathrm{n})} \mathrm{x}_{\mathrm{s}}(\mathrm{n}), \text { for }|\mathrm{e}(\mathrm{n})|>t \\
\mathrm{w}(\mathrm{n})+\frac{\widetilde{\mu^{\prime}}}{\left\|\mathrm{x}_{\mathrm{S}}(\mathrm{n})\right\|_{2}^{2}+\delta} \mathrm{e}(\mathrm{n}) \mathrm{x}_{\mathrm{S}}(\mathrm{n}), \text { otherwise }
\end{array}\right.
\end{gathered}
$$

In (17) $\tilde{\mu}$ is the step size for the logarithmic algorithm update, and $\widetilde{\mu}^{\prime}$ is the step size for the classical update. Aforementioned algorithm may be referred to as the Normalized Switching FxLogLMS (NSFxLogLMS).

For the same values of reference and error signals, the update term for each algorithm is different. In case of big error signal values the MNFxLMS algorithm has the biggest correction factor, although both the MNFxLMP and FxLogLMS algorithms update coefficients in much slower manner, what makes them more robust to impulses. Furthermore, since the update term in the proposed modification is even smaller than that for the MNFxLMP algorithm, one can impose bigger values of the step size, without worrying about the algorithm stability.

What is also worth mentioning is that for an error signal value tending to infinity both the MNFxLMS and MNFxLMP algorithms update term would also tend to infinity, whereas for the FxLogLMS and NSFxLogLMS it tends to zero. For error values close to \pm 1 , both the MNFxLMS and MNFxLMP algorithms update coefficients with constant values, whereas the FxLogLMS algorithm stops the update since $\log |e(n)| / e(n)$ is very close to 0 . Similar behavior is observed in case of the proposed modification. When magnitude of the error signal drops below 1, the algorithm becomes effectively the Normalized LMS algorithm, which updates coefficient very gently, and it is able to converge. The MNFxLMP correction term is much bigger, which may result in oscillations around optimal solution and an excessive mean square error. The FxLogLMS algorithm, on the other hand, cannot update coefficients if error value drops below 1, since logarithmic transformation tends to infinity then.

This analysis shows, that the proposed modification combines robustness of the FxLogLMS algorithm for impulses in noise and the accuracy of the FxLMS algorithm for small values of the error. Normalization of the step size improves convergence speed in both cases.

\section{SIMULATIONS}

To examine properties of the proposed algorithm in comparison to other algorithms, and the FxLogLMS algorithm in particular, simulation analysis is performed.

Acoustic paths are taken from the attachment to [2], which are considered as a benchmark for many publications on 
impulsive noise control, e.g. [4], [5], [6], [8]. The acoustic and electric paths are then modeled as FIR filters with the order of 256 for the primary path, $P(z)$, and with the order of 128 for the secondary path, $S(z)$.

Several cases for the reference signal are investigated:

- $A$ : purely impulsive noises modeled using the alpha stable distribution with $\alpha=1.3$,

- $\quad B$ : purely impulsive noises modeled using the alpha stable distribution with $\alpha=1.7$,

- $\quad C$ : mixed noise consisting of component modeled using alpha stable distribution of $\alpha=1.7$, sinusoids of frequencies $165 \mathrm{~Hz}, 290 \mathrm{~Hz}$ and $410 \mathrm{~Hz}$,

- $\quad D:$ non-impulsive multi-tonal noise consisting of sinusoids of frequencies $200 \mathrm{~Hz}, 250 \mathrm{~Hz}, 300 \mathrm{~Hz}, 450 \mathrm{~Hz}, 550$ $\mathrm{Hz}$ and $600 \mathrm{~Hz}$, distorted with random noise in $200-300 \mathrm{~Hz}$ band,

- $\quad E$ : real recorded hammering noise,

- $\quad F$ : real recorded shooting noise.

The premise for using the non-impulsive noise is that for many applications, impulses appear from time to time and they supplement a high-level acoustic floor present due to operation of other devices or device parts. Finally, real life impulsive noises are also used. Two signals of hammering and shooting noise were recorded and then their amplitude was scaled to range from $-100 t$ to $100 t$. An interesting study was to fit recorded noise to alpha stable distribution and estimate its parameters. It shows that real impulsive noises have relatively small characteristic exponent, what is not concerned in the literature. The values for both signals are presented in Table 1. Every simulation was repeated many times and obtained results were averaged. All data used for simulation are available at [15].

TABLE I. ALPHA-STABLE MODEL PARAMETERS.

\begin{tabular}{|c|c|c|c|}
\hline Real noise & $\boldsymbol{\alpha}$ & $\boldsymbol{\gamma}$ & $\mathbf{a}$ \\
\hline Hammering & 1.16 & 0.38 & -0.47 \\
\hline Shooting & 0.71 & 2.81 & 0.19 \\
\hline
\end{tabular}

Parameters for every algorithm are chosen experimentally in such way that the algorithms are stable and converge as fast as possible, providing at the same time satisfactory noise reduction. It should be stressed that for simulations the parameter $p$ can be estimated a priori, thanks to knowing the signal parameters. However, in practical applications it may not be possible. All parameters are presented in Table 2 .

For evaluating algorithms performance, the Averaged Noise Reduction (ANR) index is used, as defined in [10]:

$$
\begin{gathered}
\operatorname{ANR}(n)=20 \log _{10}\left(\frac{A_{e}(n)}{A_{d}(n)}\right), \\
A_{q}(n)=\lambda A_{q}(n-1)+(1-\lambda)|q(n)|
\end{gathered}
$$

and $\lambda=0.9$

\begin{tabular}{|c|c|c|c|c|}
\hline Noise & $\begin{array}{l}\text { MN } \\
\text { FxLMS }\end{array}$ & $\begin{array}{l}\text { MN } \\
\text { FxLMP }\end{array}$ & $\begin{array}{l}\text { FxLog } \\
\text { LMS }\end{array}$ & $\begin{array}{l}\text { NSFx } \\
\text { LogLMS }\end{array}$ \\
\hline$\alpha=1.3$ & $\tilde{\mu}=1 \cdot 10^{-4}$ & $\begin{array}{c}\tilde{\mu}=3 \cdot 10^{-4} \\
\mathrm{p}=1.2\end{array}$ & $\tilde{\mu}=2 \cdot 10^{-5}$ & $\begin{array}{l}\tilde{\mu}=7 \cdot 10^{-3} \\
\tilde{\mu}^{\prime}=7 \cdot 10^{-5}\end{array}$ \\
\hline$\alpha=1.7$ & $\tilde{\mu}=5 \cdot 10^{-4}$ & $\begin{array}{c}\tilde{\mu}=1 \cdot 10^{-3} \\
\mathrm{p}=1.6\end{array}$ & $\tilde{\mu}=6 \cdot 10^{-5}$ & $\begin{array}{l}\tilde{\mu}=2 \cdot 10^{-2} \\
\tilde{\mu}^{\prime}=2 \cdot 10^{-4}\end{array}$ \\
\hline mixed & $\tilde{\mu}=4 \cdot 10^{-4}$ & $\begin{array}{c}\tilde{\mu}=1 \cdot 10^{-3} \\
p=1.6\end{array}$ & $\tilde{\mu}=6 \cdot 10^{-5}$ & $\begin{array}{l}\tilde{\mu}=2 \cdot 10^{-2} \\
\tilde{\mu}^{\prime}=2 \cdot 10^{-5}\end{array}$ \\
\hline non-imp & $\tilde{\mu}=4 \cdot 10^{-4}$ & $\begin{array}{c}\tilde{\mu}=1 \cdot 10^{-4} \\
p=1.9\end{array}$ & $\tilde{\mu}=1 \cdot 10^{-4}$ & $\begin{array}{l}\tilde{\mu}=1 \cdot 10^{-2} \\
\tilde{\mu}^{\prime}=1 \cdot 10^{-3}\end{array}$ \\
\hline hammering & $\tilde{\mu}=2 \cdot 10^{-4}$ & $\begin{array}{c}\tilde{\mu}=5 \cdot 10^{-3} \\
\mathrm{p}=1.06\end{array}$ & $\tilde{\mu}=5 \cdot 10^{-5}$ & $\begin{array}{l}\tilde{\mu}=1 \cdot 10^{-2} \\
\tilde{\mu}^{\prime}=1 \cdot 10^{-3}\end{array}$ \\
\hline shooting & $\tilde{\mu}=2 \cdot 10^{-4}$ & $\begin{array}{l}\tilde{\mu}=0.5 \\
\mathrm{p}=0.6\end{array}$ & $\tilde{\mu}=2 \cdot 10^{-5}$ & $\begin{array}{l}\tilde{\mu}=1 \cdot 10^{-2} \\
\tilde{\mu}^{\prime}=1 \cdot 10^{-3}\end{array}$ \\
\hline
\end{tabular}

TABLE II. ALGORITHM PARAMETERS USED FOR SIMULATIONS.

\section{A. $\quad$ SoS with $\alpha=1.3$}

For case A (see Fig. 4), the proposed SNFxLogLMS algorithm converges the fastest among presented algorithms. MNFxLMP is slower than the proposed algorithm, but faster than any other algorithms. Performance of the FxLogLMS and MNFxLMS algorithms is comparable. All algorithms show good robustness to impulses.

\section{B. $\quad S \alpha S$ with $\alpha=1.7$}

When noise tends to be less impulsive (see Fig. 5), the difference between the FxLogLMS and MNFxLMS algorithms is more visible than that in the previous case. For this noise, FxLogLMS is the slowest among the presented algorithms. The proposed SNFxLogLMS algorithm is the fastest, but its superiority over the MNFxLMP is not as visible as in more impulsive noise case.

\section{C. $\quad$ Mixed noise}

Under mixed conditions of the impulsive and nonimpulsive components in the noise, results are similar to previous cases (see Fig. 6). The MNFxLMS and FxLogLMS algorithms show almost identical performance, while the MNFxLMP algorithm is a bit faster. The SNFxLogLMS algorithm exhibits the fastest convergence rate and provides good noise reduction. 


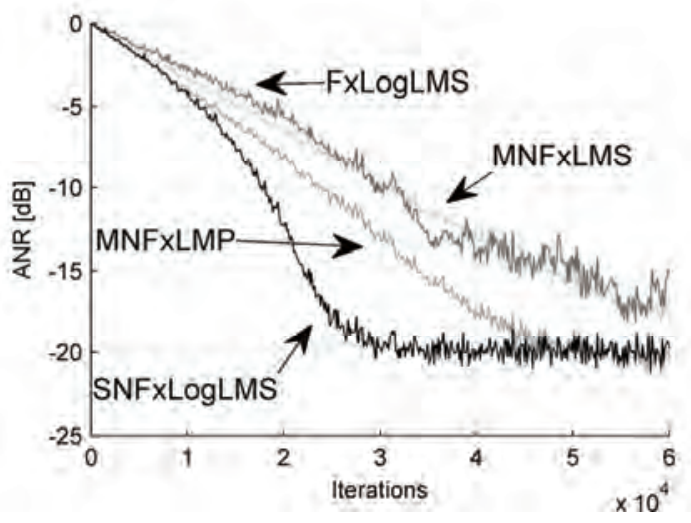

Fig. 4. ANR curves for $\mathrm{S} \alpha \mathrm{S}$ noise with $\alpha=1.3$.

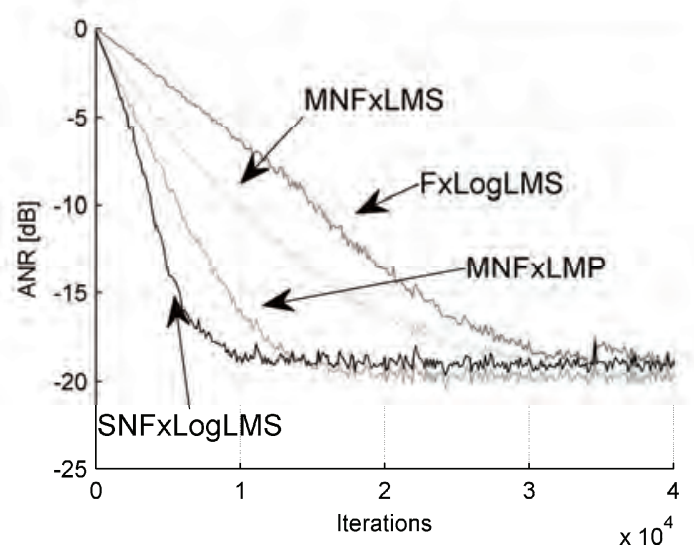

Fig. 5. ANR curves for $\mathrm{S} \alpha \mathrm{S}$ noise with $\alpha=1.7$.

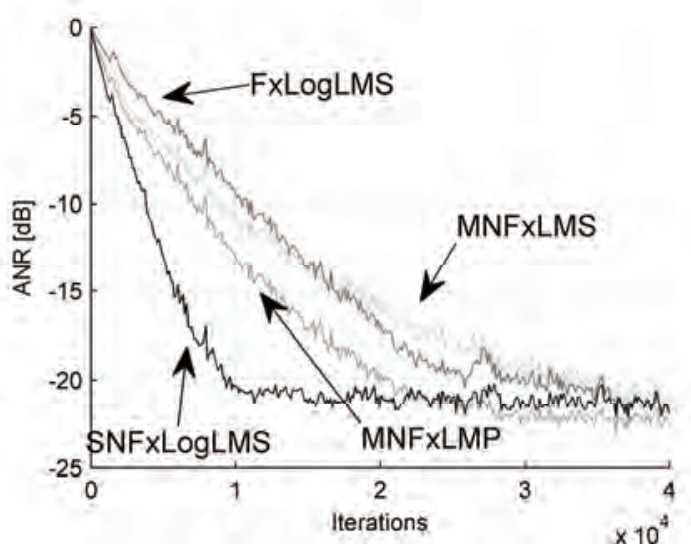

Fig. 6. ANR curves for mixed noise.

\section{Non-impulsive noise}

For the non-impulsive noise case (see Fig. 7), the FxLogLMS algorithm shows much worse performance than the other algorithms. This is caused by the logarithmic transformation, which implies stopping the adaptation for small values of the error. Performance of the MNFxLMS and proposed SNFxLogLMS algorithms is comparable. Interesting phenomenon is observed for MNFxLMP. Increasing the step-size even by a small value causes the algorithm to provide poor reduction, thus the step-size must be chosen as very small, resulting in slower convergence comparing to the MNFxLMS algorithm.

\section{E. Hammering noise}

For the real hammering noise (see Fig. 8), the MNFxLMS and FxLogLMS algorithms have almost identical performance. The same can be stated about MNFxLMP and SNFxLogLMS; however, these two algorithms converge significantly faster than the other two. On the other hand, the MNFxLMP algorithm converges much slower than that for the simulated noises and in this case even slower than the MNFxLMS and FXLogLMS algorithms. This is caused by the small value of $p$, which has to be smaller than the characteristic exponent. The proposed algorithm performs very well. It has the fastest convergence rate.

\section{$F$. Shooting noise}

The shooting noise is in turn very impulsive, with approximated characteristic exponent value around 0.7 (see Fig. 9). In such case, the MNFxLMS and FxLogLMS algorithms show very similar performance. On the other hand, the MNFxLMP algorithm converges much slower than that for the simulated noises and in this case even slower than the MNFxLMS and FXLogLMS algorithms. This is caused by the small value of $p$, which has to be smaller than the characteristic exponent. The proposed algorithm performs very well. It has the fastest convergence rate.

\section{CONCLUSIONS}

It follows from the simulations that the proposed SNFxLogLMS algorithm converges faster than other algorithms designed for impulsive noise, which is due to the normalization. Benefits of using this algorithm are particularly evident if the noise contains other, nonimpulsive high-level components. Then, it reduces them similarly as the MNFxLMS algorithm, whereas algorithms designed for the impulsive noise only yield significantly poorer performance.

For real recorded noises, the proposed algorithm shows also very good performance. It does not require a priori knowledge about the signal, what the MNFxLMP algorithm needs to have, to estimate the $\mathrm{p}$ parameter. It is worth mentioning that real impulsive noises have relatively small characteristic exponent, what is not concerned in the literature, and thus validating of algorithms for such signals is very important to recognize their true properties. 


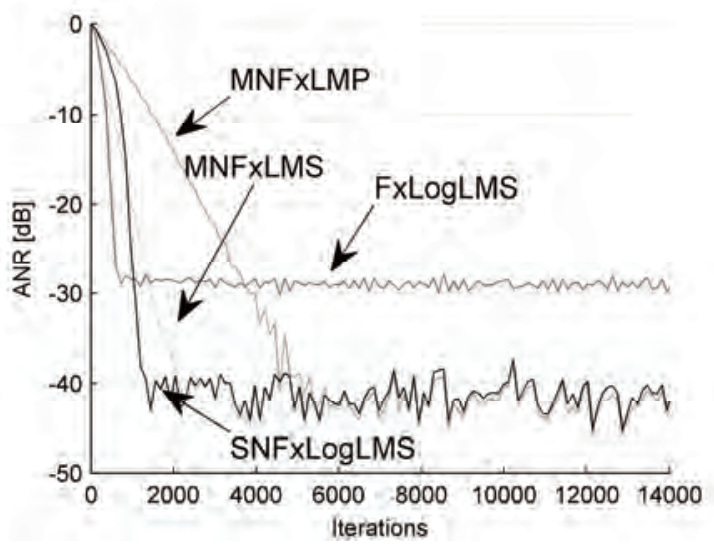

Fig. 7. ANR curves for non-impulsive noise.

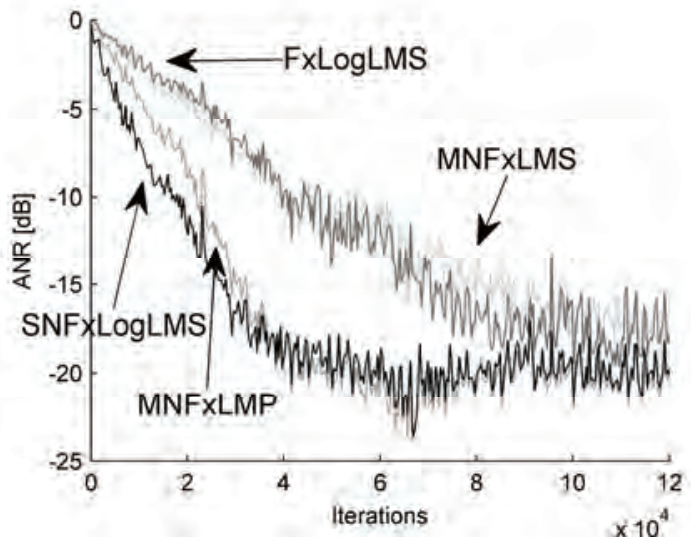

Fig. 8. ANR curves for hammering noise.

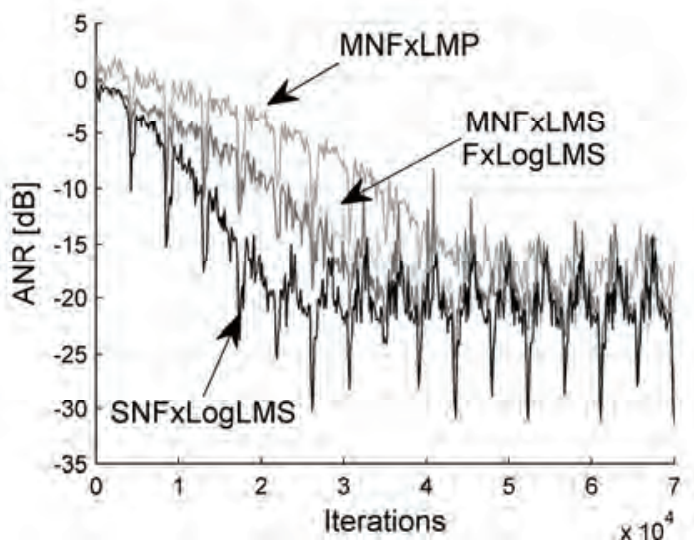

Fig. 9. ANR curves for shooting noise.

The proposed algorithm can be applied to reduce impulsive device noise by controlling vibration of the device casing [15]. Such application can mitigate one of the main problems met in active noise control, and impulsive noise control in particular, which is generation of local zones of quiet of small dimensions. The active casing, as investigations reported in [15] demonstrate is able to provide global control in the whole environment around the casing. Because this application involves a number of sensors and actuators, its efficient implementation is important, still guaranteeing high convergence rate. A modification to the LMS adaptation rule as proposed, e.g., in [17] can be then useful.

\section{REFERENCES}

[1] S. J. Elliott, Signal Processing for Active Control. London, U.K: Academic, 2001.

[2] S. M. Kuo and D. R. Morgan, Active Noise Control Systems: Algorithms and DSP Implementations. New York: Wiley, 1996.

[3] L. Wu, H. He, and X. Qiu, "An Active Impulsive Noise Control Algorithm with Logarithmic Transformation," IEEE Trans. Audio, Speech, Lang. Process., vol. 19, no. 4, pp. 1041-1044, May 2011.

[4] M. T. Akhtar and W. Mitsuhashi, "Improving robustness of filtered-x least mean p-power algorithm for active attenuation of standard symmetric- $\alpha$-stable impulsive noise," Applied Acoustics, vol. 72, no. 9, pp. 688-694, 2011.

[5] S. M. Kuo, K. Kuo, and W. S. Gan, "Active Noise Control: Open Problem and Challenges," Proc. Int. Conf. on Green Circuits and Systems (ICGCS), pp. 164-169, Shanghai, 2010.

[6] M. T. Akhtar and W. Mitsuhashi, "A modified normalized FxLMS algorithm for active control of impulsive noise," Proceedings of EUSIPCO, pp. 1-5, Aalborg, 2010.

[7] S. C. Douglas, "A family of normalized LMS algorithms," IEEE Signal Processing Letters, vol. 1, no. 3, pp. 49-51, 1994.

[8] W. Wierzchowski, "Active control of impulsive noise," M.S. thesis, Silesian Univ. of Technology, Gliwice, Poland, 2013.

[9] R. A. Niedzielski. (May 1991). Environmental impulse noise study: final report, Minnesota Pollution Control Agency. [Online]. Available: http://www.nonoise.org/library/impulse/impulse.htm_.

[10] M. T. Akhtar and W. Mitsuhashi, "Improving performance of FxLMS algorithm for active noise control of impulsive noise," J. Sound and Vibration, vol. 327, no. 3-5, pp. 647-656, 2009.

[11] X. Sun, S. M. Kuo, and G. Meng, "Adaptive algorithm for active control of impulsive noise," J. Sound and Vibration, vol. 291, no. 1-2, pp. 516$522,2006$.

[12] R. Leahy, Z. Zhou, and Y. C. Hsu, "Adaptive filtering of stable processes for active attenuation of impulsive noise," in Proc. IEEE ICASSP'95, May 1995, vol. 5, pp. 2983-2986.

[13] M. T. Akhtar, W. Mitsuhashi, "Improved adaptive algorithm for active noise control of impulsive noise", Proc. ICSP 2008, pp. 2669-2672, Beijing, October 2008.

[14] M. Pawelczyk, "Active noise control - a review of control-related problems," Archives of Acoustics, vol. 33, no. 4, pp. 413-424, 2008.

[15] S. Wrona, M. Pawelczyk: "Controllability-oriented placement of actuators for active noise-vibration control of flexible structures using memetic algorithms", Archives of Acoustics, vol. 38, no. 4, 2013, pp. 529-536.

[16] S. Wrona, M. Pawelczyk: "Active reduction of device multi-tonal noise by controlling vibration of multiple walls of the device casing", Proc. International Conference on Methods and Model in Automation and Robotics - MMAR, IEEE, 2014, pp. 687-692.

[17] D. Bismor: "LMS algorithm step size adjustment for fast convergence", Archives of Acoustics, vol. 37, no. 1, 2012, pp. 31-40. 